\title{
Développer des compétences orales à l'université : quels modèles et quelles pratiques de référence?
}

Developing Oral Skills at the University: Which Models and Reference Practices?

\section{Caroline Facq-Mellet}

\section{OpenEdition}

Journals

Édition électronique

URL : https://journals.openedition.org/recherchestravaux/4590

DOI : 10.4000/recherchestravaux.4590

ISSN : 1969-6434

Éditeur

UGA Éditions/Université Grenoble Alpes

Édition imprimée

ISBN : 978-2-37747-326-7

ISSN : 0151-1874

Référence électronique

Caroline Facq-Mellet, « Développer des compétences orales à l'université : quels modèles et quelles pratiques de référence? », Recherches \& Travaux [En ligne], 99 | 2021, mis en ligne le 08 décembre 2021, consulté le 11 décembre 2021. URL : http://journals.openedition.org/recherchestravaux/4590 ; DOI : https://doi.org/10.4000/recherchestravaux.4590

Ce document a été généré automatiquement le 11 décembre 2021.

(C) Recherches \& Travaux 


\title{
Développer des compétences orales à l'université : quels modèles et quelles pratiques de référence?
}

Developing Oral Skills at the University: Which Models and Reference Practices?

\author{
Caroline Facq-Mellet
}

1 La réflexion que nous proposons se situe dans le cadre de la mise en place d'un enseignement consacré à l'exercice de la parole orale à l'université de Paris-Nanterre et conçu comme une alternative à la pratique de l'éloquence au sein de cet établissement depuis plusieurs années via l'association Eloquentia ${ }^{1}$. Cette pratique est elle-même à replacer dans le contexte plus général de l'évolution de l'université et de ses missions depuis la réforme de Bologne qui inscrit nettement la dimension professionnelle dans l'enseignement universitaire. La maîtrise de l'oral apparaît ainsi comme une compétence essentielle pour l'insertion professionnelle. Or, l'université privilégie encore très souvent la pratique de genres de l'écrit ou de genres de l'écrit oralisé. Néanmoins, si ceux-ci peuvent sembler adaptés pour une fonction évaluatrice à grande échelle, ils se révèlent en partie inappropriés lorsqu'il s'agit d'acquérir une réelle aisance à l'oral. La mise en place de la réforme Licence-Master-Doctorat (LMD) à l'échelon national commence à partir de $2002^{2}$; à l'université de Nanterre, elle se déploie à partir de l'année 2006 puisque cet établissement fait partie de la vague $\mathrm{D}^{3}$. L'établissement se trouve alors confronté à la question du développement de compétences professionnelles, dont l'oral, au sein du système d'enseignement. Au-delà même des modalités d'insertion de l'acquisition de ces compétences dans le cursus universitaire de premier cycle, se pose la question du type d'oral que l'université propose de faire acquérir, en particulier lorsqu'elle favorise le développement de l'éloquence chez les étudiants. Comment, sous quelles formes et pour quels objectifs se met en place l'enseignement de ce type d'oral à l'université de Nanterre? Après avoir brièvement situé le contexte de son enseignement dans cet établissement, nous analyserons la pratique de l'éloquence telle qu'elle s'y déploie en interrogeant le modèle de l'oral qu'elle véhicule, les compétences qu'elle cherche à développer, ainsi 
que la représentation de l'argumentation qu'elle produit. Cela nous permettra d'établir un bilan critique, qui amènera à présenter le dispositif que nous avons élaboré, en le plaçant dans la perspective d'une fonction de l'oral au service de la construction des savoirs.

\section{Le contexte universitaire de l'enseignement de l'oral à l'université de Nanterre}

2 On note au sein de cet établissement une préoccupation croissante pour la maîtrise de l'oral, comme en témoigne le récent projet So Skilled (« Soft skills, expériences étudiantes et prérequis tout au long de la vie »), lauréat en 2017 de l'appel à projet Nouveaux Cursus à l'Université (NCU) du troisième Programme d'Investissements d'Avenir (PIA3), qui place la question de l'oral comme l'une des compétences fondamentales à acquérir pour tous les étudiants. Sont en effet visées, dans le projet, l'aisance à l'oral, la confiance en soi et la créativité des étudiants, comme susceptibles d'accroître leur capacité à s'insérer dans le monde professionnel. Christophe Brechet, responsable du projet So Skilled et vice-président en charge de la formation et de la vie universitaire à l'université Paris-Nanterre, souligne la dimension professionnelle recherchée par la mise en place de ce projet dans une interview accordée à Studyrama, le $1^{\mathrm{er}}$ avril 2019 :

Il va permettre aux étudiants de développer leurs compétences transversales ou soft skills afin de faciliter leur insertion professionnelle : les capacités d'analyse, de synthèse, la résolution de problèmes complexes, la créativité, l'esprit d'initiative, la confiance en soi, la capacité à travailler en groupe, à convaincre, etc. ${ }^{4}$

La notion de soft skills est d'ailleurs elle-même empruntée au domaine du management et de l'entreprise. Elle désigne globalement des compétences transversales et comportementales, de plus en plus valorisées dans le domaine professionnel ${ }^{5}$. Pourtant, cette préoccupation et cet engouement pour l'expression orale sont en décalage avec les modèles et les pratiques réelles à l'université, qui continuent à être marqués par une culture de l'écrit, aussi bien dans les modalités d'enseignement que dans l'évaluation. Le témoignage que nous pouvons apporter est partiel et donc partial dans la mesure où il aurait dû être complété par une enquête globale prévue puis annulée en raison de la crise sanitaire. Néanmoins, le constat que nous faisons est le suivant. Dans certaines formations, on prend l'oral comme objet d'études (les interactions orales en sciences du langage, par exemple) sans apprendre à le pratiquer. L'oral qui est en usage chez les étudiants dans le cadre des apprentissages est un oral formalisé, relevant davantage de l'écrit oralisé que spontané et qui prend la forme d'exposés, de plus en plus souvent accompagnés de dispositifs tels que les PowerPoint. Dans cette configuration, l'oral apparaît comme un soutien, un accompagnement de l'écrit. Ces pratiques se réfèrent au modèle magistral de l'enseignant, monologal et très formalisé. Alors que les écrits font de plus en plus l'objet d'un apprentissage par des modules de méthodologie insérés dans la formation, l'oral, même dans les formes académiques de l'exposé, semble être considéré comme devant être acquis spontanément, par l'imitation des modèles magistraux.

4 De plus, même si la didactique universitaire se développe depuis quelques années, comme en témoigne par exemple la revue RIPES (Revue internationale de pédagogie de l'enseignement supérieur), les recherches sur la didactique de l'oral à ce niveau d'étude sont encore trop peu nombreuses. Ainsi, la plupart des travaux exposés dans cette 
revue portent sur des processus généraux comme le tutorat ou la coopération ou bien ils interrogent de manière préférentielle les écrits estudiantins. On relève très peu d'articles sur la didactique de l'oral ${ }^{6}$. Même s'il fait l'objet d'interrogations de la part des enseignants, il ne semble pas encore véritablement pensé comme devant faire l'objet d'un apprentissage. À l'université de Nanterre, il est encore soit pratiqué en marge de l'enseignement officiel (comme dans les formations proposées par l'association Eloquentia), soit mis en place à titre expérimental (comme c'est le cas pour notre atelier). La dernière maquette issue de la mise en place de LMD4 intègre néanmoins un module intitulé "Orator» qui propose un enseignement centré sur les compétences rhétoriques et partiellement consacré à l'oral. Le modèle sous-jacent est celui de la rhétorique classique et les genres enseignés sont les genres délibératif et épidictique qui peuvent sembler inadéquats pour l'apprentissage d'un oral académique. En somme, on constate qu'il y a un véritable désir d'oral mais l'université est encore à la recherche de modèles de son enseignement. C'est dans ce contexte que s'est développée la pratique de l'éloquence à l'université de Nanterre, à partir de l'année 2014, par le biais de l'association Eloquentia.

\section{L'apprentissage de l'éloquence à l'université}

5 Pour l'étude de l'oral véhiculé par l'association, nous nous sommes appuyée à la fois sur son programme, disponible sur internet, mais également sur l'observation de plusieurs séances proposées au sein de l'université dans le cadre de la préparation des concours d'éloquence. Le modèle de l'oral que cette association véhicule peut se lire dans son programme pédagogique, qui comprend cinq modules : expression scénique, rhétorique classique, slam et poésie, technique vocale et respiration et enfin aspiration personnelle et professionnelle. Quelques caractéristiques peuvent être dégagées. Il s'agit tout d'abord d'un oral essentiellement monologal: la référence explicite à la rhétorique classique dans l'un des modules de la formation renvoie au modèle de l'orateur et à l'acquisition des techniques permettant de composer et de dire un discours monogéré :

Cette matière a pour objectif de comprendre les différentes structures du discours,

de comprendre les techniques de l'argumentaire, de s'entrainer à porter un

discours et enfin de développer une culture de l'échange, du dialogue et du débat ${ }^{7}$.

Des formes dialogales comme le dialogue et l'échange sont bien mentionnées mais elles n'apparaissent pas devoir faire l'objet d'un apprentissage spécifique dans les techniques de l'oral. La prédilection pour les formes monogérées tend à valoriser de manière inégale les pôles de la communication. Ainsi, le pôle émetteur apparaît comme dominant et actif, le pôle récepteur comme passif, même si la pédagogie proposée s'appuie sur le développement de la capacité d'écoute au service de la prise de parole. Ce fonctionnement tend à véhiculer une représentation simplifiée et dépassée de la communication orale comme la transmission d'un message par un pôle émetteur vers un pôle récepteur qui doit le décoder. De plus, même s'il y a une part d'improvisation, il s'agit d'un oral formel, en partie écrit, puisque, comme l'explique le programme pédagogique, la performance orale «s'appuie sur un travail d'écriture et d'organisation de la réflexion ". Il ne s'agit pas de l'oral des interactions ordinaires, ni même de celui des interactions en salle de classe. Les aspects les plus caractéristiques de l'oral tels qu'ils ont été décrits par les spécialistes comme Claire Blanche-Benveniste ${ }^{8}$ (inachèvements, entassements d'éléments paradigmatiques sur l'axe syntagmatique, 
allers et retours sur l'axe syntagmatique, etc.), sans être absents dans les réalisations attestées sont le plus possible gommés, en raison, en particulier, du travail d'écriture en amont de la production orale. Il s'agit d'un apprentissage qui vise une production brillante, littéraire, en tout cas incitant à l'utilisation des procédés formels de la rhétorique. Les références de l'association penchent d'ailleurs davantage vers la dimension artistique de l'oral, puisqu'elle convoque des genres comme la poésie, le rap, le stand-up ou encore le slam. Notons d'ailleurs que la référence au théâtre est importante. Le module d'expression scénique vise en particulier le jeu corporel. Le module de rhétorique classique accorde également une place importante à l'adaptation du corps à l'argumentation. On retrouve ici la partie de l'actio de la rhétorique classique :

La rhétorique classique induit également un travail spécifique sur la gestuelle et l'oralité dans le cadre d'un discours: le regard, le mouvement des mains, le placement de la voix, l'occupation de l'espace... Lorsqu'il s'agit de convaincre ou de prendre la parole, notre corps tout entier est convoqué 9 .

Enfin, l'apprentissage conçoit la prise de parole comme une performance, c'est-à-dire un oral unique, avec un investissement subjectif important, dans le sens où la dimension créative prend le pas sur l'aspect routinier et répétitif de la production orale. L'enseignement est tout entier tendu vers les représentations lors des différentes étapes des concours d'éloquence.

8 Ces caractéristiques de l'oral sont associées au développement de deux compétences essentielles. Il s'agit tout d'abord de favoriser l'aisance orale et le développement personnel. De ce point de vue, l'objectif est en parfaite adéquation avec ceux du projet So Skilled. D'ailleurs, le programme fait explicitement référence aux soft skills, que la pratique de l'éloquence doit développer comme vecteur de la connaissance de soi :

L'aspiration personnelle permet aux jeunes de s'interroger sur les traits de leur personnalité, leurs motivations. Le formateur amène les élèves à s'interroger sur leurs «soft skills» (compétences comportementales) et points forts/points faibles, tels que la créativité, la prise d'initiative, l'empathie, le travail en équipe, la communication $^{10}$.

Il s'agit de développer cette compétence au service d'une exposition et d'une promotion de soi. De ce point de vue, les concours d'éloquence peuvent être appréhendés comme des moments où cette présentation doit être la plus aboutie. Il s'agit bien d'une représentation dans le sens d'une construction fictive de l'orateur, un ethos scénique incarnant quelques valeurs essentielles. Si l'empathie, la bienveillance et la collaboration sont affirmées comme fondamentales dans le programme de l'association, la production orale effective est avant tout perçue et encouragée comme individuelle et centrée sur soi. Elle incarne essentiellement des valeurs comme l'effort, la sélection par l'excellence, le dépassement de soi.

Cette centration de l'apprentissage sur le pôle locutif est associée à la dimension professionnelle et à la préoccupation de l'insertion sociale, que l'on retrouve dans la valorisation, au sein du monde professionnel, des soft skills. Ainsi, parmi les matières enseignées, et à côté de l'expression scénique, la rhétorique classique et l'expression vocale, on trouve un enseignement appelé " aspiration personnelle et professionnelle " qui met en relation la maîtrise de l'oral avec la préparation des élèves à des situations d'oral professionnel ou universitaire : 
Il s'agit là de préparer les élèves à des formes de prise de parole spécifiques auxquelles ils sont susceptibles d'être confrontés : entretien d'embauche, entretien de stage, oraux scolaires, présentation de mémoire ${ }^{11}$.

11 La maîtrise de l'oral sert donc de manière privilégiée l'insertion sociale. On retrouve la relation qui s'établit entre cette pratique de l'oral et l'évolution des missions de l'université incluant une préoccupation professionnelle.

12 En ce qui concerne le modèle argumentatif véhiculé par la formation pédagogique et les concours d'éloquence, il peut être décrit comme la mise en présence de deux positions antagonistes (pour/contre) sous le regard d'un tiers qui, éclairé par l'argumentation de chacun des camps, peut déterminer une position, une opinion. Nous pouvons faire plusieurs remarques relatives à ce modèle. Tout d'abord, il s'agit du modèle judiciaire, mais également en partie d'une forme de modèle délibératif parlementaire, qui permet une prise de décision à partir de positions antagonistes. Remarquons que dans ces modèles, l'argumentation précède de peu l'action, sous forme de vote ou de jugement. Elle doit entraîner l'adhésion et avoir des répercussions immédiates, ce qui peut favoriser le développement d'une argumentation fondée sur l'exploitation des preuves subjectives au détriment du logos. De plus, la recherche de l'adhésion se fait d'une manière globale, complète et définitive, au terme d'une seule prise de parole lors d'une joute verbale. La métaphore du combat suggère qu'il s'agit de vaincre et d'éradiquer la pensée opposée et non de co-construire avec l'autre la meilleure position possible en vue d'une prise de décision. Enfin, il s'agit d'une argumentation qui valorise le pôle émetteur puisque toutes les ressources de l'argumentation lui sont confiées. La dimension personnelle, qu'elle soit fondée sur le talent ou l'effort, est valorisée.

En résumé, les concours d'éloquence s'insèrent parfaitement dans cette évolution des missions de l'université avec des compétences orales au service de l'insertion professionnelle et de la promotion de soi. Nous rejoignons de ce point de vue les remarques et les critiques qui ont été faites par Olivier Barbarant dans un article publié dans Le Monde diplomatique sujet de ces concours. La pratique véhicule un modèle de rhétorique tourné vers le pôle émetteur et s'éloignant de sa dimension citoyenne et collective :

Expression des sans-voix ou maîtrise du verbe dominant, les deux font la paire, avec pour argument clé de la part des organisateurs comme des candidats, les impératifs qu'impose une société de communication: y trouver un travail, et même sa place comme citoyen passerait par la capacité à (se) dire ${ }^{12}$.

Pourtant, si l'on considère que l'une des missions essentielles de l'université continue d'être l'acquisition de connaissances scientifiques, on peut se demander si c'est cet oral-là qu'il convient de favoriser et d'enseigner.

\section{Quel enseignement de l'oral pour la construction des savoirs?}

\section{Un autre modèle de l'argumentation}

Le dispositif que l'on a cherché à mettre en place part donc d'une réflexion critique de l'enseignement de l'éloquence à l'université tel qu'on a pu l'observer. Il s'agit moins d'ailleurs de condamner les pratiques de concours d'éloquence que de comprendre les enjeux auxquels ils répondent et de proposer, de manière complémentaire, un autre 
type d'enseignement, avec d'autres compétences, d'autres représentations de l'oral et d'autres modèles argumentatifs. Ce que l'on cherche à développer est avant tout une pratique argumentative orale au service de la construction des savoirs scientifiques. Le modèle sous-jacent à cet objectif comporte plusieurs traits dominants. Tout d'abord, il s'agit d'un oral polygéré, fondé sur l'hypothèse générale d'une prééminence de l'interaction dans le développement des compétences orales argumentatives et cognitives. Cette importance accordée aux interactions verbales est maintenant bien partagée au sein de l'enseignement primaire et secondaire ${ }^{13}$. Celles-ci sont considérées comme favorisant la décentration et la construction de l'objet de savoir par la négociation du sens. L'autre n'est pas perçu comme simple récepteur mais il est associé étroitement à la production langagière et à l'élaboration des savoirs. Dans ce contexte, on cherche à développer un oral qui accorde de l'importance aux deux pôles, et dans lequel ces pôles non seulement s'échangent constamment mais co-construisent les meilleurs arguments en vue de la conclusion la plus valide possible. De ce fait, l'investissement personnel n'est pas spécifiquement valorisé ; il s'agit avant tout d'un oral de groupe, dans lequel la co-énonciation est au fondement du développement de la pensée et du raisonnement. Le modèle ne récuse pas l'aide de la routinisation des pratiques et porte son attention moins sur le pôle émetteur que sur le discours luimême.

Il s'agit ensuite d'un oral situé. Même si la question de l'aisance est importante, il s'agit principalement de développer non une compétence transversale mais de viser précisément l'oral académique en relation avec la discipline enseignée; ni conversationnel, ni oratoire, cet oral ne récuse pas les spécificités de la langue orale mais il est en partie formalisé dans la mesure où il se fait selon les contraintes propres à l'enseignement et en adaptation constante avec les spécificités de la formalisation scientifique: clarté et précision de l'argumentation, emploi des termes spécialisés, renvoi à des sources scientifiques valant comme preuves, etc. Il s'agit avant tout de développer des compétences argumentatives en lien avec l'objet d'apprentissage. En sciences du langage, par exemple, il s'agira de questions propres aux enseignements : feront ainsi l'objet de questions argumentatives des sujets comme la relation entre pensée et langage, la dimension performative, ou des questions plus pointues sur la compréhension et l'interprétation de textes scientifiques linguistiques.

Le modèle argumentatif sous-jacent est très différent de celui qui est véhiculé par les concours d'éloquence. L'argumentation n'est pas directement actionnelle car il s'agit avant tout de former et transformer des esprits, en vue de la compréhension et de l'adoption des savoirs scientifiques. Elle est en prise directe avec la dimension cognitive et le développement de la raison. On retrouve ainsi les préoccupations de chercheuses comme Ruth Webb ${ }^{14}$ qui mettent en évidence la fonction cognitive de l'argumentation. Pour elles, l'image mentale provoquée par la parole participe du raisonnement et de la construction de la pensée. La parole n'est pas le vecteur d'une pensée déjà là mais contribue à son élaboration. Dans le domaine scolaire, on rencontre la même relation, en didactique, établie entre argumentation et élaboration des savoirs. Par exemple, Baruch Schwarz et Michael Baker ${ }^{15}$ en viennent ainsi à construire le mot-valise " argumentissage " pour désigner une théorie spécifique caractérisée par deux entrées complémentaires : apprendre à argumenter et argumenter pour apprendre. De plus, il s'agit d'un modèle d'oral polygéré, qui accorde toute son importance au dialogue argumentatif: les interactions langagières modifient progressivement les 
représentations sur un objet de discours au fur et à mesure des débats. Je me réfère ici à des travaux menés au sein de l'école primaire ou secondaire, comme ceux de Marie Gausse ${ }^{16}$ ou d'Étienne Bourgeois et Mariane Frenay ${ }^{17}$, qui pourraient être mis à profit dans l'enseignement universitaire. Enfin, le modèle argumentatif est celui de la problématologie proposée par Michel Meyer, qui définit l'argumentation comme un processus de négociation de désaccords autour d'une question posée. Ces désaccords peuvent être de plusieurs ordres et de forces différentes. J'ajoute que l'argumentation, dans ce cadre, est vue davantage comme un processus plutôt que comme un résultat. La définition de Marianne Doury illustre bien cette vision de l'argumentation, qu'elle conçoit comme « un mode de construction du discours visant à le rendre plus résistant à la contestation ${ }^{18}$ ». Cette dimension processuelle est à notre sens parfaitement en adéquation avec la démarche scientifique. Ce sont ces caractéristiques de l'argumentation qui sont sollicitées dans le dispositif élaboré pour la mise en place d'un atelier d'argumentation orale à l'université.

\section{Présentation du dispositif}

\section{Les principes} tout d'abord d'un enseignement en grande partie oral, même si certaines séances s'appuient sur des documents écrits (textes scientifiques devant faire l'objet d'une interprétation par exemple) et peuvent avoir été préparées par des lectures en amont. Il nous a semblé important de mettre au cœur de cet atelier non un enseignement théorique de l'oral mais une pratique effective. Nous nous appuyons sur l'idée, développée par les didacticiens de la langue orale à l'école primaire, comme Claudine Garcia-Debanc et Isabelle Delcambre ${ }^{19}$, que c'est avant tout par l'expérimentation renouvelée et routinisée que se développent les compétences dans ce domaine. Cela oblige à repenser l'évaluation et à proposer une forme d'observation permanente de la pratique orale.

Cette question est la même qui est posée pour tous les niveaux d'enseignement lorsqu'il s'agit d'évaluer l'oral. Il s'agit d'une question difficile à résoudre car elle suppose un dispositif important permettant d'observer, de catégoriser et d'analyser les productions langagières. Pour notre part, nous avons fondé l'évaluation, d'une part sur l'observation des conduites argumentatives orales, et d'autre part sur la capacité des étudiants à analyser leurs conduites et celles de leurs pairs. On a donc décidé d'associer la pratique de l'oral à une observation de ces pratiques, par des enregistrements audio ou vidéo, mais aussi par des comparaisons avec d'autres sources orales (débats, films documentaires, etc.). Il s'agit de développer des compétences chez les étudiants en leur donnant l'occasion de pratiquer l'oral académique et en même temps en développant chez eux une forme de conscience cognitive relative à ces pratiques. Ces principes nous ont obligée à concevoir un dispositif en petits groupes, dans un atelier comportant vingt étudiants au maximum, à la fois pour encourager la prise de parole et en même temps pour faciliter l'observation et l'évaluation. Enfin, la mise en place de l'atelier associe étroitement recherche et enseignement, car il s'agit de nourrir les recherches en didactique du français à l'université, encore trop peu développées. Nous souhaitons en particulier étudier les formes et le rôle des reformulations dans l'acquisition de 
compétences argumentatives orales et développer les relations entre argumentation orale et cognition.

\section{Le programme} aspects de l'oral sont travaillés. La première partie de l'atelier porte sur des caractéristiques assez générales des interactions orales: il s'agit de faire prendre conscience de ce qui favorise et ce qui entrave les interactions et, en particulier, de ce qui favorise la progression topicale, l'élaboration de l'objet de savoir. Nous accordons ensuite trois séances, d'une part à la gestion et au maintien de l'échange par l'étayage, puis à la dimension non verbale de l'échange et enfin à la gestion du désaccord.

La deuxième partie du cours porte plus spécifiquement sur des compétences explicatives et argumentatives. Sont travaillées ainsi des pratiques spécifiques relatives à la prise en compte de l'auditoire à travers des activités discursives comme l'explication d'un point de vue tenant compte d'un point de vue différent. Est ensuite abordée la question des définitions, des redéfinitions et des reformulations qui jouent un rôle déterminant dans la compréhension et l'interprétation d'une notion scientifique. Enfin, une séance est consacrée à la gestion des preuves et en particulier, dans le cadre de l'argumentation académique, à l'utilisation des sources scientifiques. Il ne s'agit que d'un programme, qui demandera sans doute à être amendé lorsqu'il sera mis en place.

La question de l'enseignement de l'oral à l'université commence à émerger. Elle apparaît bien après les interrogations formulées dans l'enseignement primaire et secondaire, dès les années 80 , qui ont nourri des recherches importantes en didactique et en linguistique. Si une didactique de l'oral à l'université pourra se nourrir de ces travaux, elle doit comprendre les spécificités de son enseignement en les replaçant dans le contexte de l'évolution des missions de l'université, de plus en plus sollicitée pour intégrer une fonction d'insertion professionnelle dans son offre de formation. Elle doit également interroger ses propositions, en les plaçant dans le contexte, plus général encore, de l'influence subie par l'université d'un modèle managérial s'imposant depuis une quinzaine d'années, et qui importe ses valeurs et son fonctionnement. L'oral pratiqué et enseigné à l'université est perméable à cette influence, comme le montre justement l'introduction d'une forme d'éloquence, en partie détournée de sa portée citoyenne et délibérative, pour porter des valeurs de promotion de soi en vue de l'insertion professionnelle. La volonté de développer un enseignement de l'oral davantage axé sur la construction des savoirs contribue à réaffirmer la mission d'enseignement scientifique de l'université. 


\section{NOTES}

1. Nous précisons que cet enseignement devait être mis en place au deuxième semestre de l'année universitaire 2020-2021. La fermeture des universités a empêché la tenue effective de l'atelier. Les propositions que nous faisons dans cet article sont uniquement programmatiques et nous ne pouvons en faire aucune évaluation. L'atelier est maintenant programmé pour l'année universitaire 2022-2023.

2. Circulaire DES du 14 novembre 2002, relative à la mise en place du schéma Licence-MasterDoctorat (LMD).

3. La Réforme LMD s'est mise en place selon plusieurs "vagues ", correspondant à des années successives en fonction des établissements : 2003 pour la vague A, 2004 pour la vague B, 2005 pour la vague $C$ et 2006 pour la vague $D$.

4. "Les soft skills sont intéressants pour les recruteurs, en complément de la formation disciplinaire ", interview en ligne sur: <https://www.studyrama.com/formations/filieres/ universite/les-soft-skills-sont-interessants-pour-les-recruteurs-105664> [consulté le 20 septembre 2021].

5. Même si cette notion est souvent utilisée dans le monde professionnel, il existe encore très peu d'études scientifiques sur ce sujet, et pas de définition uniformisée. On peut voir néanmoins à ce sujet l'interview de F.Jarleton, "Soft skills", disponible en ligne sur le site du journal Orientactuel : <Soft skills - Orientactuel - La lettre de l'orientation (centre-inffo.fr)> [consulté le 2 juin 2021].

6. On peut néanmoins consulter l'article de M.-N. Cocton, «Évaluer une pratique innovante: essai réflexif autour de l'expression théâtrale ", Revue Internationale de Pédagogie de l'Enseignement Supérieur, $\mathrm{n}^{\circ}$ 35(2), 2018. Disponible sur: <https://doi.org/10.4000/ripes.2116> [consulté le 13 mars 2020], ainsi que celui de T. Jeanneret, «De quelques activités langagières observées lors d'un processus d'élaboration conjointe de connaissances et d'apprentissage coopératif ", Revue Internationale de Pédagogie de l'Enseignement Supérieur, 34(2), 2018. Disponible en ligne sur: <https://doi.org/10.4000/ripes.1422> [consulté le 13 mars 2020].

7. Les parcours pédagogiques. Disponible en ligne sur : <https://eloquentia.world/les-parcours/ en-pratique/> [consulté le 13 mars 2020].

8. C. Blanche-Benveniste, Approches de la langue parlée en français, Ophrys, Gap-Paris, France, 1997.

9. Eloquentia, "Les parcours pédagogiques ", matière "Rhétorique classique ». Disponible en ligne sur : <https://eloquentia.world/les-parcours/en-pratique/> [consulté le 13 mars 2020].

10. Idem., matière « Aspiration personnelle et professionnelle ».

11. Idem.

12. O. Barbarant, «L'éloquence ou le bagou?», Le Monde diplomatique, $1^{\mathrm{er}}$ novembre 2018. Disponible en ligne sur: <https://www.monde-diplomatique.fr/2018/11/BARBARANT/59243> [consulté le 13 mars 2020].

13. On peut se référer par exemple à l'ouvrage de M. Grandaty, «Interactions et apprentissages disciplinaires : la médiation de l'enseignant ", Carrefour de l'éducation, HS n 1, 2011, p. 121-135. Disponible en ligne sur : <https://doi.org/10.3917/cdle.hs01.0121> [consulté le 20 mars 2021].

14. R. Webb, "Vision, cognition et argumentation", communication présentée à la journée d'étude du séminaire doctoral ADA « Rendre visible », Lille, 22 mai 2015.

15. B. Schwarz \& M. Baker, «Sur l'adéquation des théories de l'argumentation aux sciences de l'apprentissage et les fondements d'une théorie de "l'argumentissage" ", dans N. Muller Mirza \& C. Buty (dir.), L'Argumentation dans les contextes d'éducation, Berne, Peter Lang, 2015, p. 269-322. 
16. M. Gaussel, Développer l'esprit critique par l'argumentation: de l'élève au citoyen, dossier de veille de l'IFE, 108, Lyon, ENS de Lyon. Disponible en ligne sur : <http://veille-et-analyses.ens-lyon.fr/ DA/detailsDossier.php?parent=accueil\&dossier=108\&lang=fr $>$ [consulté le 20 mars 2021].

17. É. Bourgeois \& M. Frenay, «Apprendre en groupe. Rôle de l'asymétrie et de l'argumentation», dans C. Solar (dir.), Le groupe en formation des adultes, Louvain-la-Neuve, De Boeck, 2001, p. 99-114.

18. M. Doury, Argumentation. Analyser textes et discours, Paris, Armand Colin, coll. « Portail », 2016, p. 22.

19. C. Garcia-Debanc \& I. Delcambre, « Enseigner l'oral », Repères, $n^{\circ}$ 24-25, 2001, p. 3-21.

\section{RÉSUMÉS}

Cet article s'appuie sur une critique des concours d'éloquence au sein de l'université pour proposer d'autres pratiques de l'oral en adéquation avec la mission de construction des savoirs de cette institution de l'enseignement supérieur. L'étude s'inscrit dans le cadre d'une réflexion sur la mise en place d'un enseignement consacré à l'exercice de la parole orale à l'université de Paris-Nanterre. On y observe une demande importante pour développer de nouvelles compétences liées à la maîtrise de l'oral. Les concours d'éloquence semblent combler un vide didactique dans ce domaine. Pourtant, les modèles théoriques de ces exercices doivent être interrogés : les références sous-jacentes sont celles de la performance théâtrale ou de la joute judiciaire. Ce sont des modèles qui favorisent l'exacerbation des oppositions, la théatralisation et la prise de parole monologale. Il s'agit alors de proposer un autre enseignement dans un dispositif permettant de favoriser les interactions orales visant à la construction des savoirs scientifiques.

This article is based on a critique of eloquence contests within the University in order to propose other practices of oral expression in line with the mission of knowledge building of this institution of higher education. The study is part of a reflection on the implementation of a teaching program dedicated to the exercise of oral expression at the University of ParisNanterre. There is a significant demand for the development of new skills related to the mastery of oral expression. The eloquence contests seem to fill a didactic void in this field. However, the theoretical models of these exercises must be questioned: the underlying references are those of theatrical performance or legal jousting. These are models that favor the exacerbation of oppositions, theatricalization and monologic speech. It is then a question of proposing another form of teaching in a system that encourages oral interactions aimed at the construction of scientific knowledge.

\section{INDEX}

Mots-clés : argumentation, concours d'éloquence, soft skills, interaction orale, apprentissage Keywords : argumentation, speech contest, soft skills, verbal interaction, learning 


\section{AUTEUR}

\section{CAROLINE FACQ-MELLET}

Université Paris X-Nanterre, Modyco, UMR 7114

Caroline Facq-Mellet est maîtresse de conférences en sciences du langage à l'université de ParisNanterre. Ses travaux se situent à la croisée de l'analyse de discours, de la pragmatique et de l'argumentation. Elle travaille en particulier sur les genres de discours parlementaires depuis la Révolution française ainsi que sur l'évolution des pratiques discursives de l'institution universitaire. 